\title{
El pasaje del secundario a la universidad: un estudio longitudinal entre dos cohortes de jóvenes que egresaron de la escuela secundaria en el Area Metropolitana de Buenos Aires (AMBA)
}

Otero, Analía Elizabeth; Corica, Agustina María; Merbilhaa, Jimena

El pasaje del secundario a la universidad: un estudio longitudinal entre dos cohortes de jóvenes que egresaron de la escuela secundaria en el Área Metropolitana de Buenos Aires (AMBA)

Revista Educación, vol. 45, núm. 1, 2021

Universidad de Costa Rica, Costa Rica

Disponible en: http://www.redalyc.org/articulo.oa?id=44064134042

DOI: https://doi.org/10.15517/revedu.v45i1.41544

\section{(c) $(1) \Theta(9$}

Esta obra está bajo una Licencia Creative Commons Atribución-NoComercial-SinDerivar 3.0 Internacional. 


\section{El pasaje del secundario a la universidad: un estudio longitudinal entre dos cohortes} de jóvenes que egresaron de la escuela secundaria en el Área Metropolitana de Buenos Aires (AMBA)

Transitioning from High School to University: A Longitudinal Study of Two High School Graduate Cohorts in the Buenos Aires Metropolitan Area (AMBA)

Analia Elizabeth Otero

Programa Juventud. Facultad Latinoamericana de Ciencias Sociales (FLACSO), Consejo Nacional de

Investigaciones Cientificas y Técnicas (CONICET),

Argentina

aotero@flacso.org.ar

(iD https://orcid.org/0000-0001-6774-1434

Agustina Maria Corica

Programa Juventud, Facultad Latinoamericana de

Ciencias Sociales (FLACSO), Consejo Nacional de

Investigaciones Cientificas y Técnicas (CONICET),

Universidad Pedagógica Nacional (UNIPE), Argentina

acorica@flacso.org.ar

(iD https://orcid.org/0000-0002-4096-6841

Jimena Merbilhaa

Programa Juventud. Facultad Latinoamericana de

Ciencias Sociales (FLACSO, Argentina

jmerbilhaa@flacso.org.ar
DOI: https://doi.org/10.15517/revedu.v45i1.41544

Redalyc: http://www.redalyc.org/articulo.oa?id=44064134042

Recepción: 08 Mayo 2020

Aprobación: 11 Noviembre 2020

\section{RESUMEN:}

En el marco de la tendencia a prolongar el tiempo dedicado a las actividades educativas como una característica de la juventud en el siglo XXI, este artículo se propone reflexionar sobre la importancia de la temporalidad y el contexto como escenarios que habilitan o restringen las posibilidades de continuidad educativa en el nivel universitario. Para ello se presenta la evidencia empírica de dos estudios longitudinales realizados en los últimos 20 años, donde se reconstruyen los recorridos educativos post egreso y el pasaje del nivel secundario al nivel universitario. El objetivo del artículo es aportar al entendimiento de los recorridos educativos en el nivel universitario que trazaron dos cohortes de personas jóvenes egresadas de la escuela secundaria (1999 y 2011) que viven en el territorio del Área Metropolitana del Gran Buenos Aires y realizan estos recorridos en momentos diferentes en cuanto a contextos sociales, económicos y políticos del país. El seguimiento en el tiempo se realiza con jóvenes de distintos sectores socioeconómicos que, una vez que obtuvieron la certificación educativa del nivel medio, continuaron estudios universitarios.

Palabras ClaVe: Jóvenes, Educación Secundaria, Universidad, Estudios longitudinales, Buenos Aires.

\section{Abstract:}

Amidst the tendency to extend the time youngsters spend on educational activities as a trait of 21 st century youth, this study reflects on the importance of temporality and scenarios that enable or restrict the possibilities of educational continuity at the university level. Empirical evidence from two longitudinal studies conducted in the last 20 years is presented. Post-graduation educational routes and the transition from high school to university are reconstructed to understand university educational routes traced by two cohorts of young high school graduates (1999 and 2011) residing in the Greater Buenos Aires Metropolitan Area 
among varying social, economic and political contexts of the country. A follow-up was carried out with young people from different socioeconomic sectors who continued their university studies after receiving their High School graduation diploma.

KEYWORDS: Youth, High School Education, University, Longitudinal Studies, Buenos Aires.

\section{INTRODUCCIÓN}

La prolongación del tiempo dedicado a las actividades educativas, como una de las características de la condición juvenil del siglo XXI, se ha acoplado a la diversificación de los caminos y a la no linealidad en el trazado de itinerarios en los pasajes de la juventud a la adultez (Bendit, 2015). En base a estos preceptos, en el presente artículo se analizan los resultados obtenidos de una investigación realizada, sobre recorridos educativos en el nivel superior universitario en dos cohortes de jóvenes que viven en el Área Metropolitana de Buenos Aires (AMBA). El estudio estuvo compuesto por jóvenes que obtuvieron el título secundario y que tenían 18 años al momento de egreso. A su vez, son jóvenes que provienen de distintos sectores socioeconómicos; la muestra está compuesta por jóvenes de sectores altos, medios y bajos con base a la escuela de origen.

Asimismo, el grupo de jóvenes analizado está conformado por dos cohortes distintas, una que egreso en 1999 y otra en el año 2011, dos periodos temporales y contextuales diferentes. En este sentido, es de destacar esta variable contextual y temporal, ya que son contextos político-económicos diversos, por los que atravesó Argentina y que han incidido en los caminos educativos de estos dos grupos de jóvenes que egresaron del nivel secundario en distintas cohortes. La primera cohorte inició su transición en el marco de una de las crisis económicas más fuertes de los últimos tiempos en Argentina, con un alto desempleo juvenil y deterioro de los indicadores sociales (Maurizio, 2011).

En este marco, según un estudio antecedente ${ }^{[1]}$, se sostiene que, a fines de siglo, el origen social se impuso como factor preponderante en el despliegue de trayectorias juveniles con tendencias a la reproducción de las desigualdades de partida (Filmus, Kaplan, Miranda y Moragues, 2001). En cambio, la otra cohorte egresada en el año 2011 comenzó su recorrido por la universidad en un contexto de crecimiento y mejoría de los indicadores sociales, en conjunto a una ampliación en las oportunidades de acceso a los distintos niveles educativos (Tedesco, 2012; Kessler, 2015). En este punto es que la variación en el contexto en que estas trayectorias se desarrollan, habilita a analizar sobre la importancia de la temporalidad y el contexto como escenarios que habilitan o restringen las posibilidades de continuidad educativa en el nivel universitario.

Este artículo presenta parte de los hallazgos del proyecto Itinerarios posible o itinerarios probables: Un estudio sobre trayectorias educativas y laborales de jóvenes de distintos sectores sociales, egresados de la escuela media en Argentina (Programa de Investigaciones en Juventud, 2014-2017), que tuvo como objetivo general, indagar en las trayectorias educativas y ocupacionales de egresados de la educación secundaria de dos cohortes distintas- una de 1999 y otra de 2011-, que transitaron hacia la educación universitaria en distintos contextos económicos y que abarcaron a jóvenes que viven en la Ciudad y Provincia de Buenos Aires. A lo largo del desarrollo general de la investigación, se trabajó a partir de la combinatoria de herramientas cuantitativas y cualitativas de investigación social.

El proceso analítico se ha orientado con base en la triangulación de datos y el análisis reflexivo (Cowman, 1993). Este tipo de abordaje metodológico contribuye a profundizar en el conocimiento de las trayectorias y transiciones juveniles, y permite una aproximación más comprensiva de los fenómenos implicados. Asimismo, posibilita abarcar diferentes dimensiones, además de cotejar las situaciones en que se encuentran las personas jóvenes en distintos períodos temporales.

En este proyecto, el modelo follow-up studies (seguimiento de jóvenes que egresaron de la escuela secundaria) se aplicó a través de distintas herramientas cuantitativas y cualitativas. Por un lado, se presentan hallazgos sobre una encuesta realizada a jóvenes de las dos cohortes luego del año de egreso (2000 con 385 
casos y 2012 con 390 casos), a modo de visibilizar dónde se encontraban estudiando según el sector social de la escuela de origen inmediatamente luego del egreso. Sobre los objetivos de este artículo, para el análisis cualitativo se profundizó en los casos de una muestra intencional construida en ambos periodos sobre jóvenes que continuaron estudiando en la universidad en las dos muestras. En la muestra de la cohorte 2011, se agregó una submuestra de jóvenes que ingresaron a estudiar en las nuevas universidades del área metropolitana del Gran Buenos Aires.

Especialmente, la realización de entrevistas permitió ahondar en un abordaje cualitativo de las vivencias y recorridos que realizaron las personas jóvenes entrevistadas durante este periodo de seguimiento, con el objetivo de reconstruir transiciones juveniles en los primeros años post-egreso de la escuela secundaria, desde la propia voz de protagonistas. En particular, dadas las claves de interrogación del artículo, a lo largo de este se retoman exclusivamente los materiales derivados de dichas entrevistas, a modo de ilustrar los trayectos más usuales.

$\mathrm{Al}$ inicio del texto se presenta un marco conceptual desde donde se abordan las trayectorias juveniles actuales, señalando que la prolongación de la educación se instala como una tendencia de época. Luego, se presenta la metodología y el proyecto en el cual se enmarca este trabajo, las características de los estudios desarrollados y la categorización de las escuelas como definitorias del sector social de origen de las personas jóvenes, así como la selección de casos con base en las muestras originales de cada una de las cohortes. Luego, se recurre al análisis cualitativo a partir de las entrevistas realizadas, a modo de ilustrar la injerencia de los contextos en las posibilidades educativas de jóvenes. Posteriormente, se profundiza en el análisis de casos de la cohorte 2011 del sector bajo. Para finalizar, se expone una reflexión sobre los rasgos hallados, destacando la relevancia del contexto en la configuración de las trayectorias educativas post secundaria.

\section{MARCo TEÓRICo}

\section{Claves para analizar los recorridos educativos contemporáneos.}

Rene Bendit (2015) expone que los pasajes entre la escuela y el trabajo ya no se presentan en los clásicos y homogeneizados formatos propios de la era industrial, sino que se han heterogeneizado, y han dado lugar a múltiples y desiguales formas de vivir la juventud. En la actualidad, las transiciones que realizan las personas jóvenes no solo distan de ser catalogadas como estandarizadas para darse en forma pluralizada y extendida en el tiempo, sino que a su vez muestran combinaciones con superposición y alternancia de actividades respecto a patrones de antaño (Otero, 2011).

Sobre esta tendencia global, existe un rasgo en común respecto a los comportamientos de las personas jóvenes en los últimos 40 años. Estos, se inclinaron hacia la prolongación del tiempo dedicado a las actividades educativas y a la acumulación de años en el sistema educativo. En este sentido, la educación forma parte de las expectativas de amplios grupos sociales, lo que incita a adquirir nuevas titulaciones y credenciales (Criado, 1998).

A su vez, las trasformaciones de la modernización científico-tecnológica, así como los procesos de globalización, ha incidido en nuevos modelos de referencia en torno a la inserción laboral, así como los procesos de socialización de las nuevas generaciones. Este escenario impuso sobre la juventud, la exigencia de obtener competencias cada vez más desarrolladas y adecuadas a la demanda, donde la educación se ha convertido en la clave de la integración social, provocando consecuencias en el desarrollo biográfico y en la construcción de la propia identidad (Bendit, 2015). Este conjunto de fenómenos explica cómo la educación ha ganado terreno en la configuración de la nueva condición juvenil, al posicionarse como un factor preponderante en las transiciones e interpelando a un conjunto poblacional mayor en este siglo (Miranda, 2007). 
Aun así, ante esta extensión, masificación y fragmentación de los sistemas educativos (Buchbinder y Marquina , 2008), estudios recientes consideran que las trayectorias educativas se entienden como parte de una trayectoria social (Ezcurra, 2011; Terigi, 2014), es decir que el escenario político, social y económico de las sociedades, puede revisarse desde el trazado de trayectorias educativas a modo de reflejo de las desigualdades sociales que continúan diferenciando recorridos (Dubet y Martuccelli, 2008).

En el caso de la educación universitaria en Argentina, esta tendencia a la masificación ha sido corroborada, pero al mismo tiempo el alargamiento de las trayectorias, así como las altas tasas de abandono y bajas tasas de graduación, constituyen una problemática vigente (Chiroleu, 2018a). Asimismo, estos fenómenos se intensifican en el desempeño educativo del estudiantado que pertenece a los quintiles socioeconómicos más bajos que, según (García de Fanelli, 2015), no solo se encuentran subrepresentados entre la población universitaria, sino que logran magros resultados en términos de egreso a estas instituciones.

En el marco de estas afirmaciones, los postulados de Baudelot y Leclerq (2008) sugieren que el contexto donde se desarrollan las trayectorias, estructura las experiencias de las persoans jóvenes en su cotidianeidad, ya que la disposición hacia la continuidad que promueve o apoya una política en un contexto dado afecta la durabilidad de las trayectorias escolares (Baudelot y Leclerq, 2008). Estas lecturas sugieren que las iniciativas de la política estatal, dadas en un contexto específico, tendrían efectos en el desarrollo de las trayectorias educativas y las vivencias de jóvenes que transitan en la universidad, luego del egreso del secundario. Es en este sentido que, en el próximo apartado, se sintetizan las tendencias en la política educativa del último periodo, a modo de enmarcar las vivencias de jóvenes de las distintas cohortes.

\section{El contexto argentino y las tendencias en materia de política educativa}

En líneas generales, entrada la primera década del siglo XXI en Argentina, las ideas de universalidad se distanciaron de las lógicas de selectividad y focalización propias de los años noventa para edificar un nuevo marco de protección social integral de derechos ciudadanos garantizados por el Estado (Pautassi, 2010). Este viraje en el diseño e implementación de la política pública en general y educativa en particular, dieron cuenta, según Jacinto (2016), de los procesos de inequidad que atravesaban a las trayectorias juveniles, y principalmente se abocó al fortalecimiento del vínculo educación-trabajo, afectado por la profundización del desempleo juvenil.

En este esquema, la Ley No 26.206, Ley de Educación Nacional (Honorable Congreso de la Nación, 2006), publicada en el Boletín Oficial el 28 de diciembre de 2006 en Buenos Aires, Argentina; dispuso la obligatoriedad de la escuela secundaria, al mismo tiempo que se asistió a un proceso de extensión de las prestaciones familiares, más conocido como Asignación universal por hijo ${ }^{[2]}$, destinada a niños, niñas y jóvenes de sectores vulnerados hasta los 18 años (edad teórica de egreso del secundario).

En consonancia con estas iniciativas, la universidad pública se acercó a las personas jóvenes, ampliando las posibilidades de acceso. La expansión del mapa universitario en el segundo lustro de 2000 significó un acercamiento territorial de la universidad de gestión estatal hacia localidades que no contaban con este tipo de instituciones o tenían una oferta limitada, revirtiendo la tendencia privatista de la década anterior (Accineli, Losio y Macri, 2016). La concentración de las instituciones universitarias en los grandes centros urbanos significaba el repliegue de oportunidades de jóvenes que no contaban con los recursos para trasladarse, así como un distanciamiento de las expectativas que se construyen alrededor de este nivel educativo. La presencia federal de la universidad nacional, pública y gratuita, se alcanzó durante el periodo 2006-2015, marcando presencia en todas las provincias del territorio argentino con la creación de 23 universidades (Lucardi, 2020).

Durante 2004 -2015, el territorio del AMBA fue escenario protagónico de la ampliación institucional con la creación por ley de ocho universidades nacionales. Estos cambios en la política educativa a inicios de 
siglo, suponen algún tipo de incidencia o reversión de la tendencia de jóvenes del sector bajo, a continuar sus estudios en el nivel terciario (García de Fanelli y Jacinto, 2010).

Abonando a este contexto, el lanzamiento del Plan de Finalización de Estudios Primarios y Secundarios (FinES) para finalización de la Escuela Primaria y Escuela Secundaria a partir del año 2008 y su réplica en el año 2010, Plan de Finalización de Estudios Primarios y Secundarios 2 (FinES), tuvo alcance en todas las jurisdicciones de Argentina, ampliando las posibilidades de reingreso a las instituciones educativas, así como las posibilidades formales de iniciar un nuevo nivel post egreso de la secundaria.

El crecimiento de la política de becas estudiantiles en el nivel superior, ocupó un lugar privilegiado y acompañó este proceso de expansión. Hacia fines de 2008, la creación de Programa de Becas Bicentenario (BB) dirigido a jóvenes menores de 28 años cursantes de carreras en áreas consideradas como prioritarias y estratégicas para el desarrollo económico y productivo, se constituyó como uno de los principales programas de becas universitarias junto al Programa Nacional de Becas Universitarias (creado en 1993) (Presidenta de la Nación Argentina, 2009). A su vez, en el año 2014, el Programa de Respaldo a Estudiantes Argentinos (PROGRESAR) (Argentina Presidencia, 2014), dirigido a la continuidad educativa o formativa en jóvenes de 18 a 24 años, nació con el objetivo de incluir social, educativa y laboralmente a personas jóvenes identificadas como vulnerables, incrementando las posibilidades de acceder al sistema educativo y prolongando su paso por la educación formal desde una perspectiva proteccionista (Otero, 2019).

Además, según Chiroleu (2018b), durante el año 2012 y 2015 se implementaron 99 proyectos plurianuales en todo el país, con el objetivo de mejorar los niveles de permanencia y egreso de jóvenes que transitaban los primeros años de la educación universitaria financiados por la Secretaría de Políticas Universitarias. En este sentido, el acompañamiento y el fortalecimiento de los espacios de tutoría docente o tutoría entre pares de todas las Universidades Nacionales, fue uno de los objetivos claves de este periodo. Estas acciones gubernamentales e institucionales se traducen en la potencialidad de generar cambios en las posibilidades y formas en que jóvenes se integran a la sociedad. La predisposición de la población joven a incrementar la cantidad de años de estudio y permanecer en el sistema educativo sería uno de los efectos de estos lineamientos.

Las modificaciones que realiza la aprobación de la Ley $\mathrm{N}^{\circ}$ 27.204, Decreto Nacional 2358/2015, Ley de Implementación efectiva de la responsabilidad del Estado en el nivel de Educación Superior (El Senado y Cámara de Diputados de la Nación Argentina , 2015) publicada en el Boletín oficial el 11 de noviembre de 2015, Buenos Aires, Argentina, entra en sintonía con la sancionada Ley de Educación Nacional en el año 2006 (Honorable Congreso de la Nación, 2006). El derecho a la educación superior es concebido como bien público y como derecho humano personal y social que se articula con el desarrollo nacional y regional. A su vez, se insta al financiamiento estatal, la gratuidad del grado, el ingreso y la regularidad del estudiantado y obliga a garantizar las condiciones en el acceso, permanencia y graduación.

En un contexto de mayor redistribución y reposicionamiento del Estado (Filmus, 2017), una serie de orientaciones políticas dieron lugar a una nueva perspectiva guiada por la idea de la educación como derecho, con tendencias a la inclusión y la justicia social. Estas ideas llevadas al plano de la política educativa permiten, en teoría, el fortalecimiento de dispositivos de contención y el engrosamiento cuantitativo de jóvenes que se encuentra en condiciones de emprender y culminar los niveles educativos.

Aun así, a términos del año 2015, la llegada de una nueva alianza política llevó a cabo una agenda económica con un impacto negativo sobre las condiciones de vida de la población, ganándose la denominación de neoliberalismo tardío (Delgado y Gradin, 2017). Desde entonces, particularmente el sistema educativo fue objeto de recortes que se tradujeron en la finalización de programas, así como desmembramiento de áreas y un giro privatista de la educación (Filmus, 2017). En este sentido, la meritocracia como nueva filosofía educativa, generó un fuerte impacto sobre la política educativa, donde los esfuerzos y capacidades individuales son valorados por fuera del contexto familiar y social de donde provienen los sujetos (Filmus, 2017). 
En esta última línea cabe mencionar como ejemplo la reorientación del mencionado programa PROGRESAR. A modo de síntesis, los cambios sufridos se relacionan con la evaluación del buen desempeño escolar como clave, tanto para el acceso y la continuidad a la beca, como para obtener un plus monetario privilegiando al alumnado de mayores promedios (entre los universitarios). Esta nueva lógica que adopta el programa bajo la imposición de mayores criterios meritocráticos para la participación y continuidad promueve un acceso segmentado a la protección social, conllevando posibles riesgos en el sostenimiento de trayectorias (Otero, 2019)

Dadas estas condiciones, el análisis de las trayectorias en el tiempo luego del nivel secundario, ofrece un panorama acerca de las posibilidades que tienen las personas jóvenes respecto a la educación, a la luz de las variaciones contextuales.

\section{Metodología}

\section{El mapa de la investigación y la estrategia de seguimiento de cohortes reales}

En este artículo se analizan los hallazgos de dos proyectos de investigación, ambos con base en el Programa de Investigaciones de Juventud de la FLACSO. Por un lado, el proyecto La inserción ocupacional de los egresados de la escuela media: 10 años después (Programa de Investigaciones en Juventud, 2010-2013), financiado por la Agencia Nacional de Promoción Científica y Tecnológica PICT/2008-531, y por otro lado, su continuidad en el proyecto Itinerarios posible o itinerarios probables: Un estudio sobre trayectorias educativas y laborales de jóvenes de distintos sector sociales, egresados de la escuela media en Argentina (Programa de Investigaciones en Juventud, 2014-2017), financiado por la Agencia de Ciencia y Técnica y desarrollado en la Facultad Latinoamericana de Ciencias Sociales (FLACSO).

La estrategia metodológica utilizada, que sustenta ambos proyectos, es los estudios longitudinales, dada su capacidad en capturar los procesos que constituyen el eje de la investigación. El seguimiento de las cohortes se desarrolló durante los años 1999 hasta el año 2013, para la cohorte 1999; y desde el año 2011 hasta el año 2019 para la cohorte 2011. Las dos muestras (1999 y 2011) fueron elaboradas a partir de la selección de escuelas secundarias de localidades del AMBA. Siguiendo la tradición de los estudios del campo de la sociología de la educación, se distinguieron tres segmentos (bajo, medio, alto), tomando en cuenta los siguientes indicadores: a) infraestructura escolar; b) titulación de las y los docentes; c) titulación de los padres; d) características socioeconómicas de la población que asiste. Una vez registrados esos datos de las escuelas de la muestra, se procesaron los datos de cada persona de la población joven que integró la investigación, para categorizarlos en los estratos socioeconómicos identificados.

La técnica de seguimiento de egresados/as se basó en un modelo follow-up studiet y se utilizaron distintas herramientas de recolección de datos, tanto cualitativas como cuantitativas. Para el registro de los acontecimientos que hacen al desarrollo biográfico de actores, se utilizaró, tanto encuestas autoadministradas y presenciales, durante el último año de secundario, así como encuestas telefónicas durante los primeros años de la transición. Como corolario de las investigaciones, se realizó entrevistas a profundidad, a jóvenes de una muestra. La muestra de entrevistas en ambas cohortes se confeccionó a partir de los datos individuales relevados en el campo, hecho que permitió la homologación entre los datos cuantitativos y cualitativos relevados.

El resultado del seguimiento de cohortes reales, permitió la confección de una base de datos (microdatos) que recupera las biografías juveniles con una vasta cantidad de información sobre distintos momentos del desarrollo de las trayectorias, hecho que permite identificar procesos estructurales e individuales en el desarrollo de los acontecimientos. 
El seguimiento de la cohorte 1999 se realizó desde el año del evento del egreso del secundario (1999), año en el que se aplicó una encuesta autoadministrada y presencial a 594 personas estudiantes, todas ellas transitando el último año de la escuela secundaria. Las escuelas que formaron parte de la investigación fueron 18, todas se ubicaron en el territorio del AMBA y fueron categorizadas en segmentos socioeconómicos, según los criterios anteriormente nombrados. Al año siguiente del egreso (2000), a partir de una encuesta telefónica, se recabó información sobre las primeras actividades que realizaron las personas jóvenes durante el post egreso, concretando 385 casos.

En el año 2011, se realizó un nuevo rastreo telefónico sobre esta misma cohorte y en el año 2013 se confeccionó una muestra de entrevistas en profundidad para la cual se seleccionó intencionalmente a 30 jóvenes en seguimiento. A modo de representar los recorridos más usuales registrados en la última encuesta telefónica (2011), estos se distribuyeron en jóvenes que a. estudian b. estudian y trabajan, c. trabajan d. no trabajan y no estudian. La muestra de 30 entrevistas en su conjunto, quedó conformada por 12 jóvenes de sector bajo, 10 de sector medio y 8 de sector alto; donde 12 son mujeres y 18 hombres. En cuanto a su lugar de residencia, 12 habitaban en la Ciudad de Buenos Aires y 18 habitaban en otros partidos del AMBA.

En este artículo en particular, se presentan y analizan solo los hallazgos obtenidos en el año 2012 (primer rastreo telefónico y aplicación de una encuesta semiestructurada a 385 casos) y luego se tomaron las entrevistas de los y las jóvenes que continuaron estudiando en la universidad, luego del egreso del secundario en el año 2013, es decir 21 casos de 30.

Para la segunda cohorte, Cohorte 2011, el seguimiento se extendió desde el año 2011, año de egreso del secundario, hasta el año 2019. Al igual que el estudio antecesor, se construyó una muestra de jóvenes que se encontraban en el último año de la secundaria en el año 2011. En esta instancia se realizaron 538 encuestas en 19 escuelas situadas en el AMBA y categorizadas según los mismos criterios de la cohorte originaria. Continuando con la estrategia de investigación previa, durante el año 2012 y el año 2015 se realizó un relevamiento telefónico post-egreso, y en una etapa posterior del seguimiento -en el año 2016- se realizó entrevistas a profundidad a 30 jóvenes (ver Tabla 1). La muestra se construyó con base en las actividades registradas con mayor frecuencia y en referencia al mismo procedimiento utilizado para la confección de la muestra de entrevistas de la cohorte precedente. De las 30 entrevistas realizadas, 10 pertenecían al sector bajo, 13 al sector medio y 7 al sector alto. De ellas, 14 pertenecían a jóvenes que provienen de escuelas de la Ciudad de Buenos aires, 10 del Gran Buenos Aires, 4 de La Plata y 2 de una localidad del interior, al mismo tiempo que 16 son varones y 14 mujeres. 
TABLA 1

muestra de entrevistados -Cohorte 1999

\begin{tabular}{|c|c|c|c|c|}
\hline Sector & Universidad y carrera & Escuela & Nombre & LOCALIDAD DE RESIDENCIA \\
\hline \multirow{5}{*}{ SB } & UBA-Arquitectura & Técnica & ESTEBAN & Gran Buenos Aires \\
\hline & UNLA- Periodismo & Bachiller & MATIAS & Gran Buenos Aires \\
\hline & UBA-Ingenieria & Técnica & JUAN & Gran Buenos Aires \\
\hline & UBA-Nutrición & Bachiller & ANA & Gran Buenos Aires \\
\hline & UBA-Nutrición & Comercial & CECILIA & Gran Buenos Aires \\
\hline \multirow{8}{*}{ SM } & UTN-Ingenieria & Técnica & FRANCISCO & Gran Buenos Aires \\
\hline & UNLM-Ingenieria & Técnica & PEDRO & Gran Buenos Aires \\
\hline & UNLP-Periodismo & Bachiller & GASTÓN & Gran Buenos Aires \\
\hline & UTN-Ingenieria & Técnica & BERNARDO & Gran Buenos Aires \\
\hline & UNLP Periodis mo & Bachiller & Angelo & Gran Buenos Aires \\
\hline & UNLP-Psicologia & Bachiller & CLAUDIA & Gran Buenos Aires \\
\hline & UBA-Derecho & Bachiller & LEONELA & Gran Buenos Aires \\
\hline & UBA-Psicologia & Bachiller & LUCERO & Gran Buenos Aires \\
\hline \multirow{8}{*}{ SA } & UNLP-Derecho & Bachiller & TOMAS & Gran Buenos Aires \\
\hline & UBA-Ingenieria & Tecnica & GONZALO & Ciudad de Buenos Aires \\
\hline & UBA-Economia & Bachiller & LUIS & Ciudad de Buenos Aires \\
\hline & UBA-Arquitectura & Tecnica & OSCAR & Ciudad de Buenos Aires \\
\hline & UBA-Cienca política & Bachiller & DAMIAN & Ciudad de Buenos Aires \\
\hline & UNLP-Economia & Bachiller & MARCOS & Gran Buenos Aires \\
\hline & UBA-Sociología & Bachiller & JUUETA & Ciudad de Buenos Aires \\
\hline & UBA-Antropología & Bachiller & GABRIELA & Ciudad de Buenos Aires \\
\hline
\end{tabular}

uente: Elaboración propia con base en datos del Proyecto: La inserción ocupacional de los egresados de la escuela media: 10 años después (Programa de Investigaciones en Juventud, 2010-2013)

Sobre estas 30 entrevistas en el año 2019, se puso en marcha un nuevo trabajo de campo que buscó reentrevistar a jóvenes de la muestra que habían registrado continuidad en los estudios universitarios luego del egreso (21 casos de 30) (ver Tabla 2). A su vez, se incorporó en esta oportunidad a una nueva submuestra de jóvenes (10 casos entrevistados) que continuaron sus estudios en estas universidades (ver Tabla 3) con el objetivo de registrar los recorridos de jóvenes que iniciaron sus trayectorias universitarias en las universidades creadas a partir del año 2009 en el AMBA y caracterizadas por aglomerar a estudiantes de primera generación que se inician en este tipo de estudios (Accinelli, Losio y Macri, 2016). 
TABLA 2

Muestra de entrevistados - Cohorte 2011

\begin{tabular}{|c|c|c|c|c|}
\hline Sector & Universidad y carrera & Escuela & Nombre & LOCALIDAD DE RESIDENCIA \\
\hline \multirow{4}{*}{ SB } & UTN -Ingenieria & Técnica & FLORENCIA & Gran Buenos Aires \\
\hline & UTN -Ingenieria & Técnica & LUCAS & Gran Buenos Aires \\
\hline & UTN ingenieria & Técnica & CAMILA & Gran Buenos Aires \\
\hline & UNLP -Educacion fisica & Bachiller & GUIயERMO & Gran buenos Aires \\
\hline \multirow{10}{*}{ SM } & UTN -Ingenieria & Técnica & CLAUDIO & Ciudad de Buenos Aires \\
\hline & UBA- Quimica & Bachiller & MARTINA & Ciudad de Buenos Aires \\
\hline & UBA-Medicina & Bachiller & ANTONELLA & Ciudad de Buenos Aires \\
\hline & UTN ingenieria & Técnica & FABIO & Gran Buenos Aires \\
\hline & UNLP-Cine & Artistica & MAURO & Gran Buenos Aires \\
\hline & UNLP-Traductorado & Bachiller & MARTA & Gran Buenos Aires \\
\hline & UBA -Contador & Tecnica & SEBASTIAN & Gran Buenos Aires \\
\hline & UNLP -Periodismo & Agraria & HUGO & Provincia de Buenos Aires \\
\hline & UNGS -Ingenieria & Técnica & JORGE & Gran Buenos Aires \\
\hline & UNLP -Sociología & Bachiller & CRISTINA & Gran Buenos Aires \\
\hline \multirow{7}{*}{ SA } & UBA Ciencia política & Bachiller & MARIA & Ciudad de Buenos Aires \\
\hline & UBA Ingeniera & Bachiller & JAVIER & Ciudad de Buenos Aires \\
\hline & UBA Sociologáa & Bachiller & MARIA & Ciudad de Buenos Aires \\
\hline & UCA-Economia & Bachiller & RAUL & Ciudad de Buenos Aires \\
\hline & UBA-Medicina & Bachiller & JUANA & Ciudad de Buenos Aires \\
\hline & ITBA-Ingenieria & Bachiller & PABLO & Ciudad de Buenos Aires \\
\hline & UBA -Veterina ria & Bachiller & LORENA & Ciudad de Buenos Aires \\
\hline
\end{tabular}

Fuente: Elaboración propia con base en datos del Proyecto: Itinerarios posible o itinerarios probables:

Un estudio sobre trayectorias educativas y laborales de jóvenes de distintos sectores sociales,

egresados de la escuela media en Argentina (Programa de Investigaciones en Juventud, 2014-2017).

TABLA 3

Submuestra de entrevistados - Cohorte 2011.

\begin{tabular}{|c|c|c|c|c|}
\hline \multirow{7}{*}{ UNAJ-Ingenieria en Petroleo } & Técnica & CLARA & Gran Buenos Aires \\
\cline { 2 - 5 } & UNAJ-Trabajo Social & Bachiller & EMILSE & Gran Buenos Aires \\
\cline { 2 - 5 } & UNA Enfermeria & Bachiller & MORA & Gran Buenos Aires \\
\cline { 2 - 5 } & UNQUI- UNA Bioquimica & Técnica & SOFIA & Gran Buenos Aires \\
\cline { 2 - 5 } & UNAJ -Enfermeria & Bachiller & BIANCA & Gran Buenos Aires \\
\cline { 2 - 5 } & UNDAV-Derecho & Comercial & DANIEL & Gran Buenos Aires \\
\cline { 2 - 5 } & UNDAV-Enfermeria & Bachiller & ANDREA & Gran Buenos Aires \\
\cline { 2 - 5 } & UNM-Economia & Bachiller & LAUTARO & Gran Buenos Aires \\
\cline { 2 - 5 } & UNM -Economia & Bachiller & CRISTIAN & Gran Buenos Aires \\
\cline { 2 - 5 } & UNM-Economia & Técnica & SANTIAGO & Gran Buenos Aires \\
\hline
\end{tabular}

Fuente: Elaboración propia con base en datos del Proyecto: Itinerarios posible o itinerarios probables:

Un estudio sobre trayectorias educativas y laborales de jóvenes de distintos sectores sociales,

egresados de la escuela media en Argentina (Programa de Investigaciones en Juventud, 2014-2017).

Todos ellos, al igual que los y las jóvenes de la cohorte 2011, registran su egreso del secundario ese mismo año y son residentes del AMBA (6 mujeres y 5 varones). La pertenencia sobre el origen socioeconómico se 
realizó a partir de una búsqueda de jóvenes que cumplieran con los criterios de segmentación aplicados, de esta forma este grupo de 10 entrevistados fueron categorizados como parte del sector bajo.

Para este artículo, se presentan los datos sobre la encuesta al primer año de egreso (2012) que muestra un panorama de las elecciones educativas post egreso sobre 390 casos para luego a partir de distintos fragmentos que surgieron del trabajo de campo dar cuenta de la forma que asumieron y fueron vivenciados esos recorridos.

Con base en lo explicado, se logró recolectar información a modo de consecución de secuencias y experiencias a partir de datos que le dan sentido a la experiencia vivida por los sujetos (Sautu, 2004). Frente a la complejidad que las transiciones entre la educación y trabajo han asumido en la actualidad, este tipo de estudios longitudinales permiten la reconstrucción de los recorridos en el tiempo, así como el registro de las primeras actividades juveniles y las distintas modalidades de transición (Casal, García, Merino y Quesada, 2006). En este sentido es que el contexto se torna relevante para comprender el escenario en el que desarrollaron los distintos eventos.

En síntesis, para este artículo se analizaron datos cuantitativos que emergen de una primera encuesta telefónica al año de egreso de ambas de cohortes a modo de mostrar las primeras elecciones de los y las jóvenes, como punto de partida de sus recorridos. Luego se analizan los datos cualitativos que emergen de entrevistas realizadas a la muestra de jóvenes en estudio (en la cohorte 1999 el análisis se a lo largo de 14 años de egreso; y para la cohorte 2011, el análisis se realiza a lo largo de 8 años de este mismo evento).

La confección de las muestras y el armado de categorías organizadas en tres segmentos socioeconómicos, permite indagar en los recorridos educativos que se dieron en dos momentos económicos, políticos y sociales distintos en un mismo territorio. Particularmente para los objetivos de este artículo, se analizan las trayectorias educativas que registraron haber continuado estudiando en la educación superior universitaria, luego del egreso del secundario.

\section{Discusión de los datos: iguales pero diferentes, trayectorias de personas universitarias en contexto}

Como se ha sostenido en apartados precedentes, en las últimas décadas, la extensión del tiempo transcurrido en el sistema educativo junto al proceso de masificación, configuraron una tendencia en ascenso, que impactó en la condición juvenil a nivel regional y global, que conformó nuevas trayectorias juveniles, al mismo tiempo que interpeló los discursos y expectativas de los sujetos (Bendit, 2015; Criado, 1998). Este fenómeno propuesto en el primer apartado y en investigaciones que anteceden a este trabajo (Corica y Otero, 2017; Miranda y Corica, 2018), se reconfirma en la muestra en estudio. En efecto, al analizar los datos sobre las actividades que se encontraban haciendo las personas jóvenes luego del egreso del secundario, se encuentra que en su mayoría ambas cohortes tendieron a permanecer en el sistema educativo formal.

Al observar las tendencias generales entre ambas cohortes, la posibilidad de acceder a la educación superior universitaria luego del primer año de egreso de la escuela secundaria, aparece con mayor prominencia en la cohorte 2011 en comparación con la cohorte 1999 (ver Tabla 4). 
TABLA 4

Distribución porcentual de estudiantes de las cohortes 1999 y 2011, según dónde están estudiando al $1^{\circ}$ año de egreso, por sector social de la escuela

\begin{tabular}{|c|c|c|c|c|c|c|}
\hline \multirow{2}{*}{ Dónde están estudiando } & \multicolumn{3}{|l|}{2000} & \multicolumn{3}{|l|}{2012} \\
\hline & Bajo & Medio & Alto & Bajo & Medio & Alto \\
\hline Universidades & $48,2 \%$ & $79,1 \%$ & $91,2 \%$ & $78,2 \%$ & $73,0 \%$ & $89,6 \%$ \\
\hline Institutos Terciarios / Profesorados & $24,1 \%$ & $8,2 \%$ & $7,8 \%$ & $10,9 \%$ & $13,5 \%$ & $4,20 \%$ \\
\hline $\begin{array}{l}\text { Institutos de Formación Profesional/ } \\
\text { Cursos }\end{array}$ & & & & & & \\
\hline Total & $100,0 \%$ & $100,0 \%$ & $100,0 \%$ & $100,0 \%$ & $100,0 \%$ & $100,0 \%$ \\
\hline
\end{tabular}

Fuente: Elaboración propia con base en datos del Proyecto: Itinerarios posible o itinerarios probables: Un estudio sobre trayectorias educativas y laborales de jóvenes de distintos sectores sociales, egresados de la escuela media en Argentina (Programa de Investigaciones en Juventud, 2014-2017).

Se puede verificar que, mientras los grupos del sector medio y alto especifican la tendencia hacia la continuidad educativa de nivel superior como modalidad transicional preeminente en ambos períodos, el estudiantado del sector bajo acrecienta significativamente su acceso a las instituciones universitarias en el último tiempo.

Este cambio se da en detrimento de las elecciones por la educación terciaria o cursos que primaba en las posibilidades de las personas jóvenes de la cohorte 1999. Los cambios observados posicionan al contexto como una variable desde donde explicar estas preferencias. En este sentido, y como se sostuvo en los apartados anteriores, el avance en la protección social y políticas de promoción de la continuidad educativa, presentan un escenario donde continuar estudiando dentro del sistema universitario aparece como deseable y posible para el conjunto de jóvenes de la muestra (Corica, 2015). La proliferación de nuevas universidades públicas denota un aumento de matrícula, posible de observar en los grandes centros urbanos como el Gran Buenos Aires (Unzué, 2019) y que se corrobora en el comportamiento del sector bajo que se emplaza en esos territorios. Además, como se ha observado en el segundo apartado, la emergencia de distintos programas y políticas promotoras de la continuidad educativa han incorporado a los sectores subalternos desprovistos por largo tiempo de este derecho (Chiroleu, 2018a). De esta forma es posible aseverar, como se adelantó al inicio del texto, que el contexto donde se desarrollan las trayectorias estructura las experiencias de las personas jóvenes y afecta las posibilidades en el acceso, así como la durabilidad de las trayectorias escolares (Baudelot y Leclerq, 2008).

Ante esta situación inicial, la herramienta cualitativa permitió identificar formatos de continuidad en los estudios superiores universitarios que se han denominado como: trayectorias de continuidad lineal.

Los recorridos lineales o trayectorias de continuidad lineal, se caracterizan por el trazado de trayectorias donde el evento del egreso se vislumbra próximo, alcanzable según la planificación del estudiantado. Estos comportamientos predominan en jóvenes universitarios del sector alto en la cohorte 1999 y se replican en los recorridos del mismo sector de jóvenes de la cohorte 2011. Este grupo continuó estudiando inmediatamente, luego del egreso del secundario y en su mayoría lograron graduarse en tiempo y forma o se encontraban muy cerca de lograrlo en el tiempo estipulado. Marcos ejemplifica esta modalidad de transición; luego de egresar de una escuela secundaria de la Ciudad de La Plata en el año 1999, se recibió de economista en la Universidad Nacional situada en esa misma ciudad hacia el año 2007. Durante sus años como estudiante universitario trabajó en la empresa de su padre solo durante el cese de actividades académicas, abocándose a tiempo completo a sus estudios. En este plano, la dedicación a tiempo completo a los estudios indica que el trayecto educativo no se encuentra en una relación de competencia con el mundo del trabajo, esfera que ha sido entendida como un tensor de las actividades educativas juveniles (Ibarrola, 2005). Me dijo (el padre): 
'vos estudiá lo que vos quieras; yo me voy a encargar de que consigas trabajo'; bueno, listo pero él no quería que trabajara durante la carrera (Marcos ${ }^{[3]}$, comunicación personal, 13 de noviembre de 2013).

Cuatro meses antes de graduarse, el joven ingresó como pasante en un banco internacional. Al momento de la entrevista, en el año 2013, se encontraba trabajando en el mismo banco en un puesto gerencial y había sido becado para realizar un master en finanzas en una universidad privada, desplegando una trayectoria lineal.

Casos similares se repiten en la cohorte 2011. Por ejemplo, Lorena vive en la Ciudad de Buenos Aires, es una joven hija de padres universitarios, se inscribió en la carrera de veterinaria, dedicándose solo al estudio los primeros cinco años, para adentrarse a realizar tareas remuneradas en el ámbito profesional de su incumbencia, durante el último tramo de la carrera. Cuando se le preguntó por el tiempo que le llevó la cursada, comentó que tenía planificado egresarse hacia el año 2018- es decir en tiempo y forma- pero que este evento fue aplazado por un año ya que estuvo participando de una beca de intercambio en Francia durante ese año. Esta beca se trató de una experiencia de formación preprofesional dirigida a estudiantes avanzados/ as con posibilidades de acreditar dicha experiencia en el plan de estudios, motivo por el cual la entrevistada aplicó.

Al momento de la entrevista, en el año 2019, se encontraba rindiendo los últimos finales de su carrera, había acumulado experiencia en su profesión y también, a partir de la experiencia, reforzó su perfil profesional y ámbito de inserción laboral deseado a futuro Cuando supe que me iba a ir de intercambio me relajéporque sabia que iba a perder un año entre comillas... me iba sumar (Lorena ${ }^{[4]}$, comunicación personal, 5 de noviembre de 2019). En este punto se considera que, si bien estos aplazamientos son usuales en la mayoría de los recorridos universitarios, los motivos del alargamiento invitan a reflexionar sobre sus efectos en los trayectos educativos, claramente si bien su egreso fue postergado, el momento del egreso continuaba siendo una certeza para la entrevistada y, en efecto, a su vuelta se encontraba finalizando el recorrido; en este sentido se considera como parte de los trayectos de continuidad lineal.

A contrapartida de estos recorridos que se han denominado como lineales, jóvenes del sector bajo que iniciaron sus trayectorias en el nivel universitario muestran una diversidad de situaciones. Por un lado, jóvenes de la cohorte 1999, muestran salidas abruptas del nivel universitario que se asemejan a los comportamientos que asumen las personas jóvenes del sector medio de esta misma muestra. Por ejemplo, Cecilia, comenzó a estudiar la carrera de Nutrición en la Universidad de Buenos Aires, relata que la diferencia en la exigencia entre la escuela secundaria y la universidad, sumado a la distancia entre su hogar y la facultad, la llevó a poner punto final a su corta experiencia universitaria. Sin culminar su primer cuatrimestre; se inscribió en un magisterio del cual se egresó a los 21 años: noté que en ese paso de la secundaria a la facultad hay una brecha, que falta un poco más (Cecilia[5], comunicación personal, 2 de septiembre de 2013). Este relato es usual entre otros jóvenes del sector bajo, donde la universidad aparece como ámbito lejano, sin mediaciones ni dispositivos de acompañamiento, que den soporte a las desigualdades educativas, según el colegio de origen, propias de la segmentación educativa.

Jóvenes de este mismo sector y también del sector medio, egresados de la misma cohorte (1999), comentan una situación similar, con la diferencia que la finalización de la educación terciaria significó un paso previo sobre el cual sostener una posible trayectoria universitaria. Es decir, que culminar los estudios terciarios y la rápida inserción laboral a partir del ejercicio de la docencia, les permitió pensar en la posibilidad de comenzar una carrera universitaria a posterior. En ambos sectores se puede distinguir que las personas jóvenes toman decisiones respecto a las circunstancias, soportes y el contexto socioeconómico, lo que da lugar a la formación de trayectorias más inestables y erráticas. No obstante, en la cohorte 2011, el comportamiento de jóvenes del sector bajo cobró características específicas, que se detallarán a continuación. Como adelanto, se sostiene que un nuevo contexto de ampliación de políticas educativas, en el marco de la extensión de un sistema de protección social, aportó recursos para el sostenimiento de los trayectos universitarios, dando lugar a la emergencia de recorridos que se ha denominado Trayectorias de continuidad con perseverancia. 


\section{ACCEDER Y PERSEVERAR}

La herramienta cualitativa permitió identificar formatos distintos de continuidad en los estudios superiores universitarios. La estrategia metodológica y su abordaje, permitió observar diferencias en la forma en que jóvenes delinean sus trayectorias universitarias, abonando a la idea de que la democratización de estas instituciones, muestra complejidades (Chiroleu, 2018b).

Si bien las personas jóvenes de los distintos sectores sociales iniciaron sus trayectorias en casas de altos estudios, la gran diferencia que se sitúa entre los caminos universitarios de jóvenes del sector bajo de la cohorte 2011 y sus pares del sector alto, es que, a contramarcha de la linealidad en los estudios que sostienen los grupos más privilegiados, el grupo de jóvenes del sector bajo muestran trayectos educativos extendidos en el tiempo, con avances y estancamientos, así como momentos de abandonos y vueltas al estudio, donde la idea de previsibilidad o inminente fecha de egreso se desdibuja: algún día me voy a recibir (Florencia ${ }^{[6]}$, comunicación personal, 12 de agosto de 2019).

Este fenómeno observado se ha denominado Trayectorias de continuidad con perseverancia y resulta un comportamiento novedoso y propio de esta cohorte. Es decir, estas personas jóvenes acceden a la universidad, pero no logran cumplir en el tiempo estipulado por los programas de estudio en tiempo y forma. La carrera que en términos formales llevaría 5 - 6 años, dependiendo de la disciplina elegida, se torna indefinida y sujeta a los avatares de la vida que atraviesan. En este punto entran en juego los recursos, prioridades y decisiones. A su vez, el grupo de jóvenes del sector medio parecen inclinarse a una modalidad de trayectoria lineal, propia de los sectores acomodados, en este sentido los recursos económicos familiares y el acceso a trabajos de tiempo parcial aparecen como sostenedores de carreras universitarias para esta nueva cohorte.

A modo de ilustrar los recorridos de perseverancia observados en el conjunto de jóvenes de los sectores bajos, se seleccionaron historias que dan cuenta del retraso y dilación en los tiempos de estudio en el marco de circunstancias que tensionan las actividades educativas y que se interponen con el rendimiento académico. A su vez, reflejan la importancia que le dan a su trayectoria universitaria y el aporte de la política educativa en el sostenimiento de estos trayectos.

De las entrevistas a profundidad, se halló que continuar los estudios en la universidad es posible para el conjunto de la muestra, es decir que logran acceder, pasar los exámenes y constituirse en personas estudiantes afiliadas y adaptadas (Piedrahita y Mendoza, 2010). Por ejemplo, el caso de Florencia se trata de una estudiante inmigrante egresada de una escuela técnica del sur del conurbano. En sus relatos se hace presente la idea de ampliación de oportunidades, a partir de la gratuidad de la universidad y la política orientada a la inclusión educativa. En su narración existió un patrón continuo al expresar que el estudio abre posibilidades de progreso y de trabajo donde se juega el futuro.

Esta vivencia es reforzada por familiares que se muestran como el contraejemplo: mi mamá, aparte, siempre me hablaba también de que tenia que seguir, de que tenía que... que no haga como ella, que se quedó de ama de casa y después no consiguió trabajo en ningún lado y... y eso. Posibilidades... posibilidad de progresar, de encontrar un buen trabajo, de... de todo, básicamente. (Florencia ${ }^{[7]}$, comunicación personal, 12 de agosto de 2019). Desde esta perspectiva, el continuar estudiando se encuentra ligado a oportunidades de trabajo profesional y progreso económico. Las promesas del retorno de la educación parecen seguir vigentes en el imaginario de este sector social, con aspiraciones de movilidad social ascendente.

Florencia mantuvo una trayectoria laboral discontinua y tensionada por momentos de necesidad económica o de cuidados de familiares, desde el secundario hasta la universidad, situación que la llevó a mantener un recorrido errático de idas y vueltas. Florencia aparece en todo su relato atenta a las necesidades materiales de su madre y su hermana menor, tal es así que continuamente ayuda a su mamá en la costura con maquinaria hogareña. La actividad laboral que realiza la entrevistada resulta vital para la reproducción de la vida cotidiana de su hogar, estos ingresos definen el pago de servicios y alquiler, que queda sujeto a la cantidad de producción que madre e hija logren diariamente. Es decir que los ingresos no son generados solamente 
con la motivación de sostenerse individualmente y obtener dinero para gastos de la universidad y ocio como sucede usualmente en los relatos en el sector medio de esta cohorte.

En este marco, los programas educativos a los que accedió la entrevistada aparecen como una ayuda, un aporte que, si bien no logra cubrir todos los gastos que significa asistir a la universidad, es valorado por la estudiante, al igual que la infraestructura que le provee la universidad, como por ejemplo el comedor. En la última entrevista realizada en el año 2019, Florencia comenta que dejó la facultad un año, es decir desde el año 2017 hasta el año 2018. La jefa de familia se había quedado desempleada, obligando a la joven a buscar un trabajo de tiempo completo. Ese año lo dedicó a trabajar como vendedora de ropa en condiciones de informalidad laboral. A pesar de encontrase en tercer año de ingeniería y ser técnica química no accedió a ningún puesto de trabajo relacionado con su área de formación e interés, hecho que ella relaciona con la discriminación que existe en el rubro sobre las mujeres.

Esta lectura también se encuentra presente en los relatos de otras entrevistadas egresadas de la escuela técnica y se enlaza con estudios recientes que muestran la discriminación y desventajas con las que se encuentran las mujeres en el mercado de trabajo (D'alessandro, 2016). Al mismo tiempo, en línea con investigaciones precedentes, también se vincula a la importancia de las redes de contactos dispar entre los sectores socioeconómicos, para el acceso al mercado laboral en segmentos con mejores condiciones (Otero y Fridman, 2015). Es decir que las desventajas para estas jóvenes se multiplican.

En su relato se da cuenta como las tensiones económicas que en una primera etapa de transición a la universidad aparecían controladas, en el nuevo escenario post kirchnerismo se recrudecen. El desempleo de su madre y la baja en la percepción de la beca PROGRESAR a partir de los cambios hacia el año 2016 - que entre otras cuestiones exceptúa a extranjeros - resultó una combinatoria excluyente para la trayectoria universitaria de Florencia.

Hacia el año 2019, su madre volvió a incorporarse al mundo del trabajo en un autoempleo y en un diálogo entre ambas, Florencia le expresa sus ganas de volver a estudiar. Finalmente, vuelve a cursar a mediados del cuatrimestre, pidiendo favores a los profesores para que la dejen presentarse a los parciales, a pesar de que no había cumplido con el presentismo requerido por las cátedras. Su militancia estudiantil, aportó a la concesión de dichos favores y a la continuidad en el contacto con la universidad, sin embargo, perdió la mayoría de las materias de ese año por sentirse perdida. En el momento de la entrevista, vuelve a enfatizar sus deseos de recibirse, comenta como planifica año a año su carrera, que con retrasos, idas y vueltas, aún continúa como un proyecto y un deseo firme y perseverante.

Otro es el caso de Andrea, una egresada de una escuela modalidad humanística de la periferia del AMBA y partícipe de la submuestra de personas entrevistadas, que nuclea a estudiantes de las nuevas universidades del conurbano bonaerense. Ella cuenta que su idea inicial era continuar estudiando periodismo en un instituto privado de la Ciudad de Buenos Aires, frente a los costos que este trayecto implicaba, solo quedó en un deseo. Ante la incertidumbre sobre qué hacer y luego de unos meses de realizar trabajos esporádicos en tiendas de ropa, hacia el año 2012 se inscribió para ofrecer servicios dentro de la policía. No logra ser admitida dentro de la fuerza y trascurren dos años entre momentos largos de desempleo y empleos precarizados y temporales. Hacia el año 2017, y con una preocupación central por la salida laboral, define a partir de una charla entre conocidos afines a la profesión, continuar estudiando la licenciatura en enfermería que ofrece la Universidad de Avellaneda, creada en el año 2009. La cercanía, la gratuidad y la rápida salida laboral que prometía esta carrera termina por direccionar sus estudios hacia la universidad.

Desde el momento de ingreso percibió la beca PRONAFE (Programa Nacional de Formación de Enfermeros) ${ }^{[8]}$. Hacia el año 2018, comenta que los pagos se retrasaron con consecuencias en su cursada, relata por ejemplo que los días de cursada estaba todo el día sin comer o leía los textos desde el celular porque no tenía dinero para costear las copias. Hacia lo que podía, algunos profesores te mandan los apuntes vía mail, todo digitalizado y trataba de leerlo desde el celular, pero es más difícil asi (Andrea ${ }^{[9]}$, comunicación personal, 4 de octubre de 2019). Su madre percibe una pensión no contributiva para madres de siete hijos, equivalente 
a un haber mínimo y no cuenta con ingreso alternativo. Su padre se encuentra desempleado y se incomoda al hablar de su familia, mostrándose reticente a ahondar en la situación familiar. Su novio aparece en los relatos sosteniendo en parte esta trayectoria, dando a entender que pasa gran parte de la semana en la casa de su pareja.

En el año 2019, Andrea estaba tramitando su título intermedio de enfermera universitaria con la expectativa de lograr acceder a un trabajo con mayor estabilidad, aun así, desde el ingreso a la carrera, la actividad laboral había sido favorable, permitiéndole exponer en su curriculum vitae, su condición de estudiante de enfermería, hecho que la habilitó para trabajos esporádicos en asistencia a consultorios, cuidado de personas enfermas y tareas similares, relacionadas a su profesión. A futuro se imagina trabajando como enfermera, licenciarse y avanzar en la carrera de medicina en la Universidad de Buenos Aires, ansiosa por superar el temido Ciclo Básico Común.

El último caso, como el anterior, refleja la importancia del acercamiento al territorio de las nuevas universidades creadas, así como el aporte de los sistemas de becas en los recorridos de los y las jóvenes. Tal es la vivencia de Clara, una joven hija de padre y madre con secundario incompleto, que se inscribió a la carrera de ingeniería en petróleo, a partir de sentirse interpelada por la promoción de ingenieros formados en áreas estratégicas y ligados a los recursos naturales. Su militancia política barrial, que luego se extiende a la universidad, le dio acceso a información valiosa para su recorrido educativo; en este sentido, Clara accede tempranamente a la beca PROGRESAR, la cual combinó con otros estipendios que ofrecía la universidad, a modo de acuerdos con empresas que financiaban a jóvenes con promedios más altos.

Cuenta que desde que asiste a la universidad descubrió un nuevo mundo y que su hermana y su madre también comenzaron a estudiar en la misma universidad. Te decian bueno abi hay un futuro que te está esperando, entonces yo sentía que por ahi una de esas podia ser yo. (Clara[10], comunicación personal, 6 de septiembre de 2019). Aun así, durante el recorrido por la universidad, cuenta que en varias oportunidades se vio tentada por obtener un trabajo que le permitiera comprar bienes que no estaban a su alcance, ya que el acceso a becas solo le permite subsistir. Sin embargo, la promesa de egresar se impuso con mayor fuerza con la perspectiva de un trabajo como profesional. De esta forma, la entrevistada se encontraba hacia el 2019 en cuarto año de la carrera de Ingeniería, acompañada por una serie de becas que le permitían continuar su carrera sin participar en el mercado laboral.

El conjunto de casos seleccionados, refleja dos dimensiones que se encuentran presentes en la construcción de las trayectorias educativas en el nivel universitario del sector bajo de la muestra. Por un lado, características personales ligadas a la acción perseverante mediada por el deseo de egresarse de estas instituciones y, por otro lado, la importancia que asume la política estatal en el sostenimiento de estos caminos de perseverancia.

\section{Conclusiones}

Acumular años de escolaridad y continuar estudiando luego del secundario, aparece como una tendencia de época que unifica las expectativas, deseos y actividades de gran parte de la juventud en la actualidad. Distintos fenómenos han conducido a las personas jóvenes a inclinarse por el camino del estudio, marcando diferencias respecto a las trayectorias de antaño.

La desocupación, la valorización de la educación, así como los procesos de ampliación, expansión y heterogeneización del sistema universitario, aportaron a mejorar las oportunidades de estudio, incorporando nuevos grupos sociales. Particularmente, Argentina mantiene hasta la actualidad una serie de principios respecto a la gratuidad y el acceso irrestricto, que forman parte de la cultura universitaria como legado histórico. Sin embargo, más allá de estos postulados, los contextos políticos, económicos y sociales donde se realizan las transiciones juveniles, han sido leídos en este articulo como escenarios que habilitan o restringen las posibilidades de continuidad educativa en el nivel universitario.

A partir del acceso a material empírico sobre el seguimiento de estudiantes egresados/as de la escuela secundaria, se halló que, en contexto de crisis, recesión y alta desocupación juvenil, las oportunidades 
educativas tienden a la reproducción de los grupos sociales, donde el agrupamiento de jóvenes del sector socioeconómico alto, obtiene mayor ventaja respecto a la construcción de caminos universitarios lineales, continuos y con altas probabilidades de egreso en tiempo forma. Si bien esta tendencia se consolidó en las trayectorias de las personas jóvenes del mismo sector en la segunda cohorte en estudio, también se observó que a inicios de 2010, una mayor proporción de jóvenes del sector medio se inclinó por continuar estudiando en la universidad. A su vez, como dato novedoso, aparece una fuerte tendencia del sector bajo en continuar sus estudios universitarios, a contramarcha del contexto anterior.

A partir de la metodología utilizada se estableció que estos caminos educativos, que inicialmente se igualaban respecto al origen social, se diferenciaban según la forma que asumía esa trayectoria educativa. En este punto se halló que algunos y algunas jóvenes del sector bajo, desarrollaron lo que se denominó trayectorias de continuidad con perseverancia. Las características principales de este tipo de transición tienen que ver con la importancia que asume el contexto y, particularmente, la política educativa para que las personas jóvenes puedan ejercitar la perseverancia, que aún con marchas y contramarchas, posibilita el sostén de los trayectos universitarios.

Como desafío a futuro, resulta necesario profundizar cabalmente en los efectos de un nuevo contexto abierto desde los últimos tres años a la actualidad, donde la discontinuidad de una batería de políticas educativas en el nivel superior, así como un viraje de las políticas educativas hacia prédicas selectivas y meritocráticas se ha sumado a un contexto de crisis económica generalizada. En este punto hipotetiza que una retracción en el papel del Estado y la política educativa pondría en tensión el sostenimiento de este tipo de trayectorias.

\section{REFERENCIAS BIBLIOGRÁFICAS}

Accinelli, A., Losio, M. y Macri, A., (2016). Acceso, rezago, deserción y permanencia de estudiantes en las universidades del conurbano bonaerense. Debate Universitario, 5(9), 33-52. Recuperado de http://portalreviscien.uai.edu.ar/ ojs/index.php/debate-

Argentina Presidencia. (2014). POLITICAS SOCIALES, Decreto 84/2014, Créase el "Programa de Respaldo a Estudiantes Argentinos" (PROGRESAR). Boletín oficial de la República Argentina. Recuperado de https://ww w.boletinoficial.gob.ar/detalleAviso/primera/101157/20140127

Baudelot, C. y Leclercq, F. (2008). Los efectos de la educación. Buenos Aires, Argentina: Del estante.

Bendit, R. (2015). Juventud y Transiciones en un mundo globalizado. En A. Miranda (Ed.), Sociología de la educación y transición al mundo del trabajo Juventud, justicia y protección social en la Argentina contemporánea (pp. 25-51). Ciudad de Buenos Aires, Argentina: Editorial Teseo.

Buchbinder, P. y Marquina, M. (2008). Masividad, heterogeneidad y fragmentación: el sistema universitario argentino 1983-2007. Buenos Aires, Argentina: Biblioteca Nacional.

Casal, J., García, M., Merino, R. y Quesada, M., (2006). Aportaciones teóricas y metodológicas a la sociología de la juventud desde la perspectiva de la transición. Revista de Sociología Universidad Autónoma de Barcelona, (79), 21-48. Recuperado de https://ddd.uab.cat/record/13002

Chiroleu, A., (2018a). De la expansión de oportunidades al derecho a la universidad: un recorrido de un siglo desde la óptica de la representación social. Revista Latinoamericana de Educación Comparada, 9(13), 27-36

Chiroleu, A., (2018b). Democratización e inclusión en la universidad argentina: sus alcances durante los gobiernos Kirchner (2003-2015). Educação em Revista, (34), 1-26. Recuperado de http://www.scielo.br/scielo.php?script $=$ sci_arttext\&pid=S0102-46982018000100118\&lng=es\&nrm=iso

Corica, A. M. y Otero, A. E., (2017). Después de estudiar, estudio: Experiencia de jóvenes egresados de la escuela media. Población y sociedad, 24(2), 33-64.

Corica, A. M., (2015). Juventud y Futuro: las expectativas educativas y laborales de los estudiantes de la escuela secundaria. En A. Miranda (Ed.) Sociología de la educación y transición al mundo del trabajo Juventud, justicia 
Analía Elizabeth Otero, et al. El pasaje del Secundario a la universidad: un estudio longitudinal ...

y protección social en la Argentina contemporánea (pp. 91-116). Ciudad de Buenos Aires, Argentina: Editorial Teseo.

Cowman, S. (1993). Triangulation: a means of reconciliation in nursing research. Journal of advanced nursing, 18(5), 788-792.

Criado, E. M., (1998). Producir la juventud: critica de la sociología de la juventud. Madrid: Ediciones ISTMO

D'alessandro, M. (2016). Economia feminista: cómo construir una sociedad igualitaria. Ciudad de Buenos Aires, Argentina. Sudamericana.

Delgado, D. G. y Gradin A. (2017). Neoliberalismo tardío: Entre la hegemonía y la inviabilidad. En D. G Delgado y A. Gradin (Coords.) Neoliberalismo tardio, Teoria y praxis (pp.15-26). Ciudad de Buenos Aires, Argentina: FLACSO. Recuperado de https://bit.ly/2xJp750

Dubet, F. y Martuccelli, D., (1998). En la escuela. Sociología de la experiencia escolar. España: Losada.

El Senado y Cámara de Diputados de la Nación Argentina. (2015). Ley N²7.204, Decreto Nacional 2358/2015 Ley de Implementación efectiva de la responsabilidad del Estado en el nivel de Educación Superior. Boletín oficial. Recuperado de http://servicios.infoleg.gob.ar/infolegInternet/anexos/250000-254999/254825/norma.htm

Ezcurra, A. M. (2011). Masificación y enseñanza superior: una inclusión excluyente. Algunas hipótesis y conceptos clave. En N. Fernández-Lamarra y C.P.M. De Fátima (Comp.), La democratización de la educación superior en América Latina. Limites y posibilidades (pp.60-72). Buenos Aires, Argentina: EDUNTREF.

Filmus, D. (2017). Educar para el mercado. Escuela, universidad y ciencia en tiempos de neoliberalismo. Buenos Aires, Argentina: Editorial Octubre.

Filmus, D. Kaplan, C. Miranda, A. y Moragues, M., (2001). Cada vez más necesaria, cada vez más insuficiente. Escuela media y mercado de trabajo en épocas de globalización. Buenos Aires Argentina: Editorial Santillana.

García de Fanelli, A. M. (2015). La cuestión de la graduación en las universidades nacionales de la Argentina: Indicadores y políticas públicas a comienzos del siglo XXI. Propuesta Educativa, (43), 17-31.

García de Fanelli, A. y Jacinto, C. (2010). Equidad y educación superior en América Latina: el papel de las carreras terciarias y universitarias. Revista Iberoamericana de educación superior, 1(1), 58-75

Honorable Congreso de la Nación. (2006). Ley № 26.206, Ley de Educación Nacional. Boletín Oficial. Recuperado de http://www.inet.edu.ar/wp-content/uploads/2013/03/ley_de_educ_nac1.pdf

Ibarrola, M. D. (2005). Educación y trabajo. Revista mexicana de investigación educativa, 10(25), 303-313.

Jacinto, C. (2016). Protección social y formación para el trabajo de jóvenes en la Argentina reciente. Entramados alcances $y$ tensiones. Buenos Aires Argentina: IDES.

Kessler, G., (2015). Controversias sobre la desigualdad: Argentina, 2003-2013. Ciudad de Buenos Aires, Argentina: Fondo de Cultura Económica.

Lucardi, A. C. (2020). Las políticas universitarias del nuevo gobierno en Argentina: el desafío de fortalecer la democratización de la Universidad. Revista Universidades, 71(85), 81-96.

Maurizio, R., (2011) Trayectorias laborales de los jóvenes en Argentina:¿Dificultades en el mercado de trabajo o carrera laboral ascendente? Santiago de Chile, Chile: CEPAL

Miranda, A. y Corica, A., (2018). Gramáticas de la Juventud: reflexiones conceptuales a partir de estudios longitudinales en Argentina. En A. Corica, F.A. Freytes y A. Miranda. (Comp.), Entre la educación y el trabajo: la construcción cotidiana de las desigualdades juveniles en América Latina (pp. 27-50). Buenos Aires Argentina: CLACSO.

Miranda, A., (2007). La nueva condición joven: educación, desigualdad y empleo. Ciudad de Buenos Aires, Argentina: Fundación Octubre de Trabajadores de Edificios.

Otero A. y Fridman V., (2015). De estudiantes a trabajadores. Juventud y Transiciones en un mundo globalizado. En A. Miranda (Ed.), Sociología de la educación y transición al mundo del trabajo Juventud, justicia y protección social en la Argentina contemporánea (pp.169-198). Ciudad de Buenos Aires, Argentina: Editorial Teseo.

Otero, A. (2019). Políticas destinadas a las Juventudes: un análisis del Programa de Respaldo a Estudiantes de Argentina PROG.RES.AR. Revista Interamericana de educación de adultos, (1), 65-86. 
Otero. A., (2011). Las configuraciones de transiciones juveniles. Debates actuales sobre la educación y el trabajo. Revista Electrónica de Investigación Educativa, 13(2), 149-165. Recuperado de http://redie.uabc.mx/vol13no2/conten ido-otero.html

Pautassi, L., (2010). El enfoque de derechos y la inclusión social. Una oportunidad para las políticas públicas. En L. Pautassi (Organizadora), Perspectiva de derechos, politicas públicas e inclusión social. Debates actuales en la Argentina (pp. 27-66). Buenos Aires: Biblos.

Piedrahita, M. V. A. y Mendoza, M. Á. G. (2010). El “oficio" de estudiante universitario: Afiliación, aprendizaje y masificación de la universidad. Pedagogía y saberes, (33), 85-97. Recuperado de https://dialnet.unirioja.es/serv let/articulo?codigo $=5365001$

Presidencia de la Nación Argentina. (2009). Creación del Programa Nacional Becas Bicentenario para Carreras Científicas y Técnicas. Decreto Nacional 99/2009. Boletin Oficial. Recuperado de https://bit.ly/33x3Icn

Programa de Investigaciones en Juventud. (2010-2013). Proyecto de Investigación: La inserción ocupacional de los egresados de la escuela media: 10 años después. Argentina: Facultad Latinoamericana de Ciencias Sociales (FLACSO)

Programa de Investigaciones en Juventud. (2014-2017). Proyecto de Investigación: Itinerarios posible o itinerarios probables: Un estudio sobre trayectorias educativas y laborales de jóvenes de distintos sectores sociales, egresados de la escuela media en Argentina. Argentina: Facultad Latinoamericana de Ciencias Sociales (FLACSO)

Sautu, R., (2004). Elmétodo biográfico: la reconstrucción de la sociedad a partir del testimonio de los actores. Buenos Aires, Argentina: Lumiere ediciones.

Tedesco, J. C., (2012). Educación y justicia social en América Latina. Buenos Aires, Argentina: Fondo de Cultura Económica.

Terigi, F. (2014). Trayectorias escolares e inclusión educativa: del enfoque individual al desafío para las políticas educativas. En A. Marchesi, R. Blanco y L. Hernández (Coomps.) Avances y desafios de la educación inclusiva en Iberoamérica (pp.71-87). Madrid, España: OEI

Unzué, M. (2019). Una mirada sobre la educación superior en América Latina. Revista de Educación y Derecho, (19), 1-6. Recuperado de https://revistes.ub.edu/index.php/RED/issue/view/2144

\section{Notas}

[1]Cada vez más necesaria, cada vez más insuficiente. Escuela media y mercado de trabajo en épocas de globalización (Filmus et al, 2001).

[2]Cabe puntualizar que el Decreto del Poder Ejecutivo de la Nación N¹602/09 Asignación universal por hijo (AUH), (2009), es una asignación destinada a las niñas, niños y adolescentes cuyos padres/tutores/curadores estén desocupados o se desempeñen en la economía informal por un salario menor al Mínimo Vital y Móvil. Lo sustantivo de esta medida es que posibilitó ampliar el régimen de Asignaciones Familiares, incluyendo a trabajadores informales y desempleados, modificando así el esquema tradicional de cobertura de la seguridad social orientada a la persona trabajadora formal. Logró incluirse como parte del subsistema no contributivo y consolidarse como cobertura del ciclo obligatorio de educación formal hasta 18 años.

[3]Sector Alto, estudiante de Economía en una universidad pública de una ciudad de la provincia de Buenos Aires.

[4]Sector Alto, estudiante de Veterinaria en una universidad pública de la Ciudad de Buenos Aires.

[5]Sector Bajo, Estudiante de Nutrición en una universidad pública de la Ciudad de Buenos Aires.

[6]Sector Bajo, Estudiante de Ingeniería en una universidad pública del Gran Buenos Aires

[7]Sector Bajo, Estudiante de Ingeniería en una universidad pública del Gran Buenos Aires.

[8]Beca designada exclusivamente a estudiantes inscritos/as en la carrera de enfermería en Institutos de Educación Técnica Superior de Gestión Estatal y Privada o Universidades Nacionales al ser considerada como una profesión estratégica y con el objetivo de aumentar la cantidad y calidad de los enfermeros de la República Argentina. Ver https://bit.ly/2UPLheW 
Analía Elizabeth Otero, et al. El Pasaje del Secundario a la universidad: un estudio longitudinal ...

[9]Sector Bajo, estudiante de Enfermería en una universidad pública del Gran buenos Aires.

[10]Sector Bajo, estudiante de Ingeniería en una universidad del Gran Buenos Aires. 